Acta Crystallographica Section E

Structure Reports

Online

ISSN 1600-5368

\section{2-Carboxy-1-phenylethanaminium perchlorate}

\section{Xiu-Zhi Li, Hui Li and Zhi-Rong Qu*}

Ordered Matter Science Research Center, College of Chemistry and Chemical Engineering, Southeast University, Nanjing 210096, People's Republic of China Correspondence e-mail: quzr@seu.edu.cn

Received 25 April 2009; accepted 29 April 2009

Key indicators: single-crystal X-ray study; $T=294 \mathrm{~K}$; mean $\sigma(\mathrm{C}-\mathrm{C})=0.004 \AA$; $R$ factor $=0.053 ; w R$ factor $=0.110 ;$ data-to-parameter ratio $=16.4$.

In the title compound, $\mathrm{C}_{9} \mathrm{H}_{12} \mathrm{NO}_{2}{ }^{+} \cdot \mathrm{ClO}_{4}{ }^{-}$, an intramolecular $\mathrm{N}-\mathrm{H}$. . O interaction results in the formation of a sixmembered ring having a twisted chair conformation. In the crystal structure, intermolecular $\mathrm{O}-\mathrm{H} \cdots \mathrm{O}, \mathrm{N}-\mathrm{H} \cdots \mathrm{O}$ and $\mathrm{C}-\mathrm{H} \cdots \mathrm{O}$ interactions link the molecules into a network. A weak $\mathrm{C}-\mathrm{H} \cdots \pi$ interaction is also found.

\section{Related literature}

There has been an increased interest in the enantiomeric preparation of $\beta$-amino acids as precursors for the synthesis of novel biologically active compounds, see: Arki et al. (2004); Cohen et al. (2002); Zeller et al. (1965). For bond-length data, see: Allen et al. (1987).

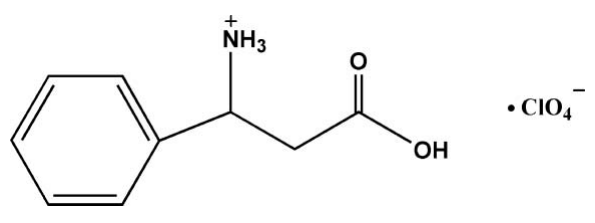

\section{Experimental}

Crystal data

$\mathrm{C}_{9} \mathrm{H}_{12} \mathrm{NO}_{2}{ }^{+} \cdot \mathrm{ClO}_{4}{ }^{-}$

$M_{r}=265.65$

Orthorhombic, Pbca

$a=6.6583(13) \AA$

$b=13.826(3) \AA$

$c=24.300(5) \AA$
Data collection

Rigaku SCXmini diffractometer

Absorption correction: multi-scan (Blessing, 1995)

$T_{\min }=0.863, T_{\max }=0.957$

21012 measured reflections 2560 independent reflections 1966 reflections with $I>2 \sigma(I)$

$R_{\text {int }}=0.058$

Refinement

$R\left[F^{2}>2 \sigma\left(F^{2}\right)\right]=0.053$

$w R\left(F^{2}\right)=0.110$

$S=1.10$

2560 reflections

156 parameters

$\mathrm{H}$-atom parameters constrained

$\Delta \rho_{\max }=0.27{\mathrm{e} \AA^{-3}}^{-3}$

$\Delta \rho_{\min }=-0.37$ e $\AA^{-3}$

Table 1

Hydrogen-bond geometry $\left(\AA,^{\circ}\right)$.

\begin{tabular}{lllll}
\hline$D-\mathrm{H} \cdots A$ & $D-\mathrm{H}$ & $\mathrm{H} \cdots A$ & $D \cdots A$ & $D-\mathrm{H} \cdots A$ \\
\hline $\mathrm{N} 1-\mathrm{H} 1 B \cdots \mathrm{O} 2$ & 0.89 & 2.13 & $2.773(3)$ & 128 \\
$\mathrm{C} 9-\mathrm{H} 9 \cdots 5^{\mathrm{i}}$ & 0.93 & 2.56 & $3.409(3)$ & 152 \\
$\mathrm{C} 3-\mathrm{H} 3 \cdots$ O5 $^{\mathrm{i}}$ & 0.98 & 2.57 & $3.370(3)$ & 139 \\
$\mathrm{C} 3-\mathrm{H} 3 \cdots \mathrm{O}{ }^{\text {ii }}$ & 0.98 & 2.58 & $3.286(3)$ & 129 \\
$\mathrm{~N} 1-\mathrm{H} 1 C \cdots \mathrm{O} 3^{\text {iii }}$ & 0.89 & 2.05 & $2.892(3)$ & 158 \\
$\mathrm{~N} 1-\mathrm{H} 1 B \cdots 3^{\text {iv }}$ & 0.89 & 2.28 & $3.046(3)$ & 144 \\
$\mathrm{~N} 1-\mathrm{H} 1 A \cdots \mathrm{O}^{\mathrm{v}}$ & 0.89 & 2.13 & $2.979(3)$ & 159 \\
$\mathrm{O} 1-\mathrm{H} 1 \cdots \mathrm{O} 2^{\text {vi }}$ & 0.82 & 2.41 & $3.046(2)$ & 135 \\
$\mathrm{O} 1-\mathrm{H} 1 \cdots 4^{\text {vii }}$ & 0.82 & 2.35 & $3.048(3)$ & 143 \\
$\mathrm{C} 8-\mathrm{H} 8 \cdots \mathrm{Cg} 1^{\text {vii }}$ & 0.93 & 2.79 & $3.688(3)$ & 162
\end{tabular}

Symmetry codes: (i) $-x+\frac{3}{2}, y-\frac{1}{2}, z$; (ii) $x-\frac{1}{2}, y,-z+\frac{1}{2}$; (iii) $x-1, y-1, z$; (iv) $x-\frac{1}{2}, y-1,-z+\frac{1}{2}$; (v) $x, y-1, z$; (vi) $x+\frac{1}{2}, y,-z+\frac{1}{2}$; (vii) $-x+2, y-\frac{1}{2},-z+\frac{1}{2}$; (viii) $-x-1, y+\frac{1}{2},-z+\frac{1}{2}$. Cg1 is the centroid of the C4-C9 ring.

Data collection: CrystalClear (Rigaku/MSC, 2005); cell refinement: CrystalClear; data reduction: CrystalClear; program(s) used to solve structure: SHELXS97 (Sheldrick, 2008); program(s) used to refine structure: SHELXL97 (Sheldrick, 2008); molecular graphics: PLATON (Spek, 2009); software used to prepare material for publication: SHELXL97.

This work was supported by the Technical Fund Financing Projects (grant Nos. 9207042464 and 9207041482) from Southeast University to ZRQ.

Supplementary data and figures for this paper are available from the IUCr electronic archives (Reference: HK2675).

\title{
References
}

Allen, F. H., Kennard, O., Watson, D. G., Brammer, L., Orpen, A. G. \& Taylor, R. (1987). J. Chem. Soc. Perkin Trans. 2, pp. S1-19.

Arki, A., Tourwe, D., Solymar, M., Fueloep, F., Armstrong, D. W. \& Peter, A. (2004). Chromatographia, 60, S43-54.

Blessing, R. H. (1995). Acta Cryst. A51, 33-38.

Cohen, J. H., Abdel-Magid, A. F., Almond, H. R. Jr \& Maryanoff, C. A. (2002). Tetrahedron Lett. 43, 1977-1981.

Rigaku/MSC (2005). CrystalClear. Rigaku/MSC, The Woodlands, Texas, USA. Sheldrick, G. M. (2008). Acta Cryst. A64, 112-122.

Spek, A. L. (2009). Acta Cryst. D65, 148-155.

Zeller, E. A., Ramachander, G., Fleisher, G. A., Ishimaru, T. \& Zeller, V. (1965). Biochem. J. 95, 262-269. 


\section{supporting information}

Acta Cryst. (2009). E65, o1214 [doi:10.1107/S1600536809016171]

\section{2-Carboxy-1-phenylethanaminium perchlorate}

\section{Xiu-Zhi Li, Hui Li and Zhi-Rong Qu}

\section{S1. Comment}

$\beta$-Amino acids are important molecules, due to their pharmacological properties. Recently, there has been an increased interest in the enantiomeric preparations of $\beta$-amino acids, as precursors for the synthesis of novel biologically active compounds (Arki et al., 2004; Cohen et al., 2002; Zeller et al., 1965). We report herein the crystal structure of the title compound.

The asymmetric unit of the title compound contains one cation and one anion (Fig.1), in which the bond lengths (Allen

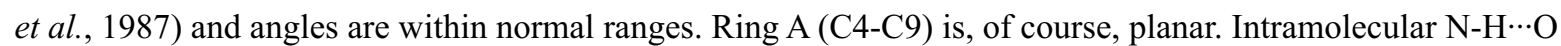
interaction results in the formation of a six-membered ring $\mathrm{B}(\mathrm{O} 2 / \mathrm{N} 1 / \mathrm{C} 1-\mathrm{C} 3 / \mathrm{H} 1 \mathrm{~B})$ having twisted conformation.

In the crystal structure, intermolecular $\mathrm{O}-\mathrm{H} \cdots \mathrm{O}, \mathrm{N}-\mathrm{H} \cdots \mathrm{O}$ and $\mathrm{C}-\mathrm{H} \cdots \mathrm{O}$ interactions (Table 1) link the molecules into a network (Fig. 2), in which they may be effective in the stabilization of the structure. There also exists a weak $\mathrm{C}-\mathrm{H} \cdots \pi$ interaction (Table 1).

\section{S2. Experimental}

Under nitrogen protection, benzaldehyde $(3.18 \mathrm{~g}, 30 \mathrm{mmol})$, malonic acid $(5.00 \mathrm{~g}, 48 \mathrm{mmol})$ and ammonium acetate $(6.00 \mathrm{~g}, 78 \mathrm{mmol})$ were added into a flask and refluxed for $10 \mathrm{~h}$ to yield a colorless precipitate. The crude product was obtained after filtration, then it was dissolved in ethanol/ perchloric acid (1:1), after slowly evaporating over a period of 4 $\mathrm{d}$, colorless prism crystals of the title compound suitable for X-ray analysis were isolated.

\section{S3. Refinement}

$\mathrm{H}$ atoms were positioned geometrically with $\mathrm{O}-\mathrm{H}=0.82 \AA$ (for $\mathrm{OH}$ ), $\mathrm{N}-\mathrm{H}=0.89 \AA\left(\right.$ for $\left.\mathrm{NH}_{3}\right), \mathrm{C}-\mathrm{H}=0.93,0.98$ and 0.97 $\AA$, for aromatic, methine and methylene $\mathrm{H}$ atoms, respectively, and constrained to ride on their parent atoms, with $\mathrm{U}_{\text {iso }}(\mathrm{H})$ $=\mathrm{xU}_{\mathrm{eq}}(\mathrm{C}, \mathrm{O}, \mathrm{N})$, where $\mathrm{x}=1.5$ for $\mathrm{OH}$ and $\mathrm{NH}_{3} \mathrm{H}$ and $\mathrm{x}=1.2$ for all other $\mathrm{H}$ atoms. 

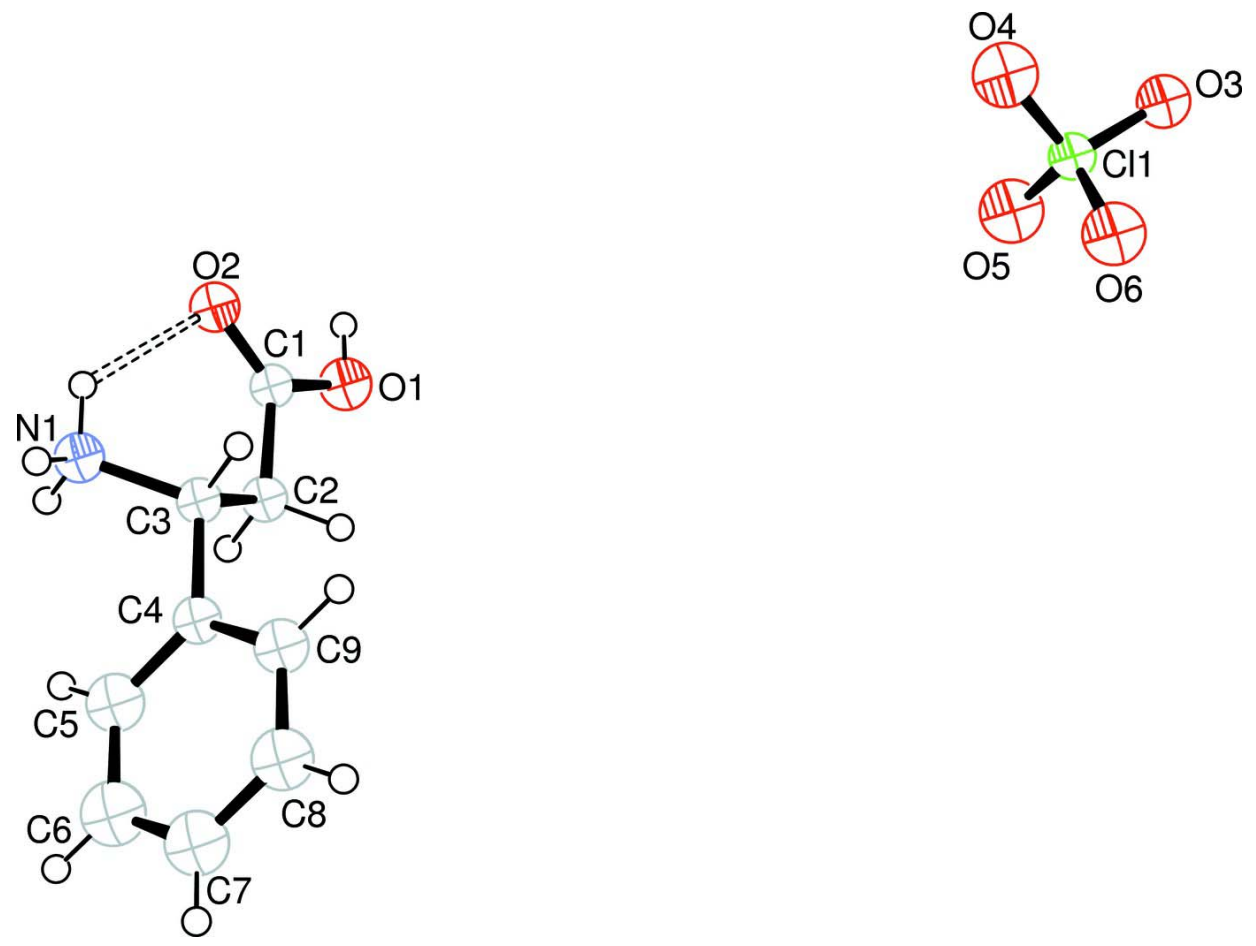

Figure 1

The molecular structure of the title molecule, with the atom-numbering scheme. Hydrogen bond is shown as dashed line.

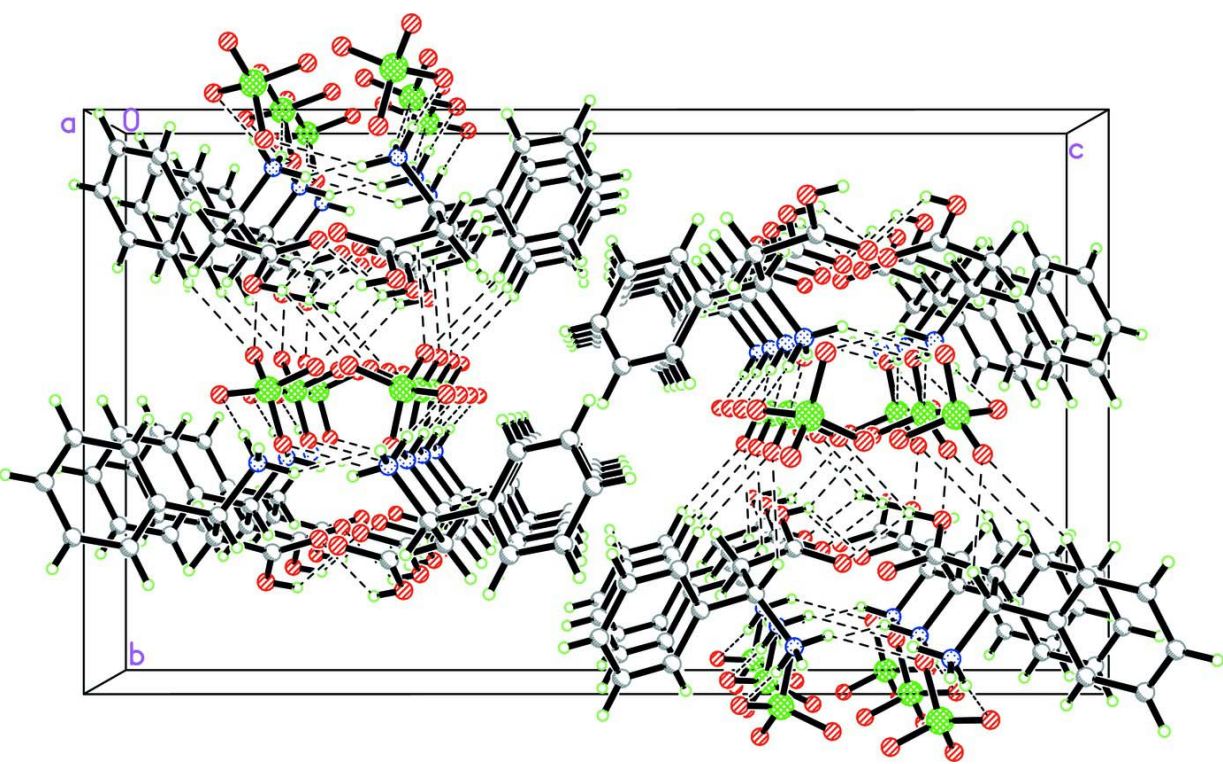

Figure 2

A partial packing diagram of the title compound. Hydrogen bonds are shown as dashed lines.

2-Carboxy-1-phenylethanaminium perchlorate

Crystal data

$\mathrm{C}_{9} \mathrm{H}_{12} \mathrm{NO}_{2}^{+} \cdot \mathrm{ClO}_{4}^{-}$

Orthorhombic, $\mathrm{Pbca}$

$M_{r}=265.65$

Hall symbol: -P 2ac 2ab 


$$
\begin{aligned}
& a=6.6583(13) \AA \\
& b=13.826(3) \AA \\
& c=24.300(5) \AA \\
& V=2237.0(8) \AA^{3} \\
& Z=8 \\
& F(000)=1104 \\
& D_{\mathrm{x}}=1.578 \mathrm{Mg} \mathrm{m}^{-3}
\end{aligned}
$$

\section{Data collection}

Rigaku SCXmini diffractometer

Radiation source: fine-focus sealed tube Graphite monochromator

CCD_Profile_fitting scans

Absorption correction: multi-scan (Blessing, 1995)

$T_{\min }=0.863, T_{\max }=0.957$

\section{Refinement}

Refinement on $F^{2}$

Least-squares matrix: full

$R\left[F^{2}>2 \sigma\left(F^{2}\right)\right]=0.053$

$w R\left(F^{2}\right)=0.110$

$S=1.10$

2560 reflections

156 parameters

0 restraints

Primary atom site location: structure-invariant direct methods
Mo $K \alpha$ radiation, $\lambda=0.71073 \AA$

Cell parameters from 1979 reflections

$\theta=3.1-27.5^{\circ}$

$\mu=0.36 \mathrm{~mm}^{-1}$

$T=294 \mathrm{~K}$

Prism, colorless

$0.45 \times 0.35 \times 0.12 \mathrm{~mm}$

21012 measured reflections

2560 independent reflections

1966 reflections with $I>2 \sigma(I)$

$R_{\text {int }}=0.058$

$\theta_{\text {max }}=27.5^{\circ}, \theta_{\min }=3.1^{\circ}$

$h=-8 \rightarrow 8$

$k=-17 \rightarrow 17$

$l=-31 \rightarrow 31$

Secondary atom site location: difference Fourier map

Hydrogen site location: inferred from neighbouring sites

$\mathrm{H}$-atom parameters constrained

$w=1 /\left[\sigma^{2}\left(F_{\mathrm{o}}^{2}\right)+(0.0351 P)^{2}+1.6729 P\right]$

where $P=\left(F_{\mathrm{o}}^{2}+2 F_{\mathrm{c}}^{2}\right) / 3$

$(\Delta / \sigma)_{\max }<0.001$

$\Delta \rho_{\max }=0.27 \mathrm{e} \AA^{-3}$

$\Delta \rho_{\min }=-0.37$ e $\AA^{-3}$

\section{Special details}

Geometry. All e.s.d.'s (except the e.s.d. in the dihedral angle between two l.s. planes) are estimated using the full covariance matrix. The cell e.s.d.'s are taken into account individually in the estimation of e.s.d.'s in distances, angles and torsion angles; correlations between e.s.d.'s in cell parameters are only used when they are defined by crystal symmetry. An approximate (isotropic) treatment of cell e.s.d.'s is used for estimating e.s.d.'s involving l.s. planes.

Refinement. Refinement of $F^{2}$ against ALL reflections. The weighted $R$-factor $w R$ and goodness of fit $S$ are based on $F^{2}$, conventional $R$-factors $R$ are based on $F$, with $F$ set to zero for negative $F^{2}$. The threshold expression of $F^{2}>\sigma\left(F^{2}\right)$ is used only for calculating $R$-factors(gt) etc. and is not relevant to the choice of reflections for refinement. $R$-factors based on $F^{2}$ are statistically about twice as large as those based on $F$, and $R$ - factors based on ALL data will be even larger.

Fractional atomic coordinates and isotropic or equivalent isotropic displacement parameters $\left(\AA^{2}\right)$

\begin{tabular}{lllll}
\hline & $x$ & $y$ & $z$ & $U_{\text {iso }} * / U_{\text {eq }}$ \\
\hline C11 & $0.99041(8)$ & $0.97917(4)$ & $0.32015(2)$ & $0.03337(17)$ \\
O1 & $0.9660(3)$ & $0.30715(14)$ & $0.32107(7)$ & $0.0428(5)$ \\
H1 & 1.0262 & 0.3182 & 0.2923 & $0.064^{*}$ \\
O2 & $0.7570(2)$ & $0.22973(13)$ & $0.26501(6)$ & $0.0365(4)$ \\
O3 & $1.0688(3)$ & $1.07288(12)$ & $0.30762(7)$ & $0.0439(5)$ \\
O4 & $0.9012(3)$ & $0.94103(16)$ & $0.27168(8)$ & $0.0630(6)$ \\
O5 & $1.1483(3)$ & $0.91952(15)$ & $0.33824(10)$ & $0.0649(6)$ \\
O6 & $0.8413(3)$ & $0.98786(15)$ & $0.36203(8)$ & $0.0584(6)$ \\
N1 & $0.4988(3)$ & $0.09957(16)$ & $0.31532(7)$ & $0.0349(5)$ \\
H1A & 0.5772 & 0.0550 & 0.3307 & $0.052^{*}$
\end{tabular}




$\begin{array}{lllll}\text { H1B } & 0.5491 & 0.1165 & 0.2828 & 0.052^{*} \\ \text { H1C } & 0.3759 & 0.0756 & 0.3107 & 0.052^{*} \\ \text { C1 } & 0.8076(3) & 0.25365(17) & 0.31064(9) & 0.0289(5) \\ \text { C2 } & 0.6999(3) & 0.22487(17) & 0.36217(9) & 0.0306(5) \\ \text { H2A } & 0.6911 & 0.2806 & 0.3863 & 0.037^{*} \\ \text { H2B } & 0.7778 & 0.1756 & 0.3810 & 0.037^{*} \\ \text { C3 } & 0.4889(3) & 0.18613(17) & 0.35177(9) & 0.0289(5) \\ \text { H3 } & 0.4139 & 0.2364 & 0.3321 & 0.035^{*} \\ \text { C4 } & 0.3749(3) & 0.16333(17) & 0.40392(9) & 0.0310(5) \\ \text { C5 } & 0.3897(4) & 0.0760(2) & 0.43014(10) & 0.0464(7) \\ \text { H5 } & 0.4766 & 0.0289 & 0.4167 & 0.056^{*} \\ \text { C6 } & 0.2752(5) & 0.0576(2) & 0.47670(12) & 0.0565(8) \\ \text { H6 } & 0.2840 & -0.0023 & 0.4940 & 0.068^{*} \\ \text { C7 } & 0.1499(4) & 0.1267(2) & 0.49722(11) & 0.0537(8) \\ \text { H7 } & 0.0739 & 0.1143 & 0.5286 & 0.064^{*} \\ \text { C8 } & 0.1367(4) & 0.2138(2) & 0.47155(11) & 0.0493(7) \\ \text { H8 } & 0.0524 & 0.2613 & 0.4857 & 0.059^{*} \\ \text { C9 } & 0.2472(4) & 0.2322(2) & 0.42480(9) & 0.0390(6) \\ \text { H9 } & 0.2352 & 0.2916 & 0.4072 & 0.047^{*}\end{array}$

Atomic displacement parameters $\left(\AA^{2}\right)$

\begin{tabular}{lllllll}
\hline & $U^{11}$ & $U^{22}$ & $U^{33}$ & $U^{12}$ & $U^{13}$ & $U^{23}$ \\
\hline C11 & $0.0277(3)$ & $0.0306(3)$ & $0.0418(3)$ & $-0.0002(2)$ & $0.0000(2)$ & $0.0054(3)$ \\
O1 & $0.0336(10)$ & $0.0582(12)$ & $0.0365(9)$ & $-0.0188(8)$ & $0.0033(8)$ & $-0.0024(9)$ \\
O2 & $0.0335(9)$ & $0.0489(10)$ & $0.0270(8)$ & $-0.0081(8)$ & $0.0018(7)$ & $-0.0040(7)$ \\
O3 & $0.0422(10)$ & $0.0332(10)$ & $0.0564(11)$ & $-0.0080(8)$ & $-0.0014(9)$ & $0.0095(8)$ \\
O4 & $0.0594(13)$ & $0.0684(14)$ & $0.0612(13)$ & $-0.0217(11)$ & $-0.0103(11)$ & $-0.0133(11)$ \\
O5 & $0.0553(13)$ & $0.0554(13)$ & $0.0841(15)$ & $0.0219(11)$ & $-0.0044(12)$ & $0.0233(11)$ \\
O6 & $0.0517(12)$ & $0.0644(13)$ & $0.0591(12)$ & $0.0037(10)$ & $0.0210(10)$ & $0.0119(11)$ \\
N1 & $0.0307(11)$ & $0.0439(12)$ & $0.0300(10)$ & $-0.0085(9)$ & $0.0019(9)$ & $-0.0045(9)$ \\
C1 & $0.0242(11)$ & $0.0291(12)$ & $0.0333(12)$ & $0.0002(9)$ & $0.0011(9)$ & $-0.0020(10)$ \\
C2 & $0.0296(12)$ & $0.0359(13)$ & $0.0262(11)$ & $-0.0056(10)$ & $-0.0013(9)$ & $-0.0024(10)$ \\
C3 & $0.0253(11)$ & $0.0355(13)$ & $0.0259(11)$ & $0.0009(10)$ & $0.0013(9)$ & $-0.0003(9)$ \\
C4 & $0.0238(11)$ & $0.0435(14)$ & $0.0256(11)$ & $-0.0039(10)$ & $-0.0005(9)$ & $0.0008(10)$ \\
C5 & $0.0485(16)$ & $0.0501(16)$ & $0.0406(14)$ & $0.0037(13)$ & $0.0071(13)$ & $0.0065(13)$ \\
C6 & $0.066(2)$ & $0.0596(19)$ & $0.0435(16)$ & $-0.0121(16)$ & $0.0047(15)$ & $0.0180(14)$ \\
C7 & $0.0425(16)$ & $0.085(2)$ & $0.0339(14)$ & $-0.0167(16)$ & $0.0088(12)$ & $0.0037(15)$ \\
C8 & $0.0388(15)$ & $0.073(2)$ & $0.0363(14)$ & $0.0045(14)$ & $0.0094(12)$ & $-0.0057(14)$ \\
C9 & $0.0323(12)$ & $0.0520(16)$ & $0.0326(12)$ & $0.0016(11)$ & $0.0003(11)$ & $0.0012(12)$ \\
& & & & &
\end{tabular}

Geometric parameters $\left(\AA,{ }^{\circ}\right)$

\begin{tabular}{llll}
\hline $\mathrm{C} 11-\mathrm{O} 5$ & $1.4067(19)$ & $\mathrm{C} 3-\mathrm{N} 1$ & $1.490(3)$ \\
$\mathrm{C} 11-\mathrm{O} 4$ & $1.421(2)$ & $\mathrm{C} 3-\mathrm{C} 4$ & $1.510(3)$ \\
$\mathrm{C} 11-\mathrm{O} 6$ & $1.4269(19)$ & $\mathrm{C} 3-\mathrm{H} 3$ & 0.9800 \\
$\mathrm{Cl1}-\mathrm{O} 3$ & $1.4297(18)$ & $\mathrm{C} 4-\mathrm{C} 5$ & $1.369(3)$ \\
$\mathrm{O} 1-\mathrm{H} 1$ & 0.8200 & $\mathrm{C} 4-\mathrm{C} 9$ & $1.373(3)$
\end{tabular}




\begin{tabular}{|c|c|c|c|}
\hline $\mathrm{N} 1-\mathrm{H} 1 \mathrm{~A}$ & 0.8900 & $\mathrm{C} 5-\mathrm{C} 6$ & $1.388(4)$ \\
\hline $\mathrm{N} 1-\mathrm{H} 1 \mathrm{~B}$ & 0.8900 & $\mathrm{C} 5-\mathrm{H} 5$ & 0.9300 \\
\hline $\mathrm{N} 1-\mathrm{H} 1 \mathrm{C}$ & 0.8900 & $\mathrm{C} 6-\mathrm{C} 7$ & $1.363(4)$ \\
\hline $\mathrm{C} 1-\mathrm{O} 2$ & $1.205(3)$ & $\mathrm{C} 6-\mathrm{H} 6$ & 0.9300 \\
\hline $\mathrm{C} 1-\mathrm{O} 1$ & $1.313(3)$ & $\mathrm{C} 7-\mathrm{C} 8$ & $1.359(4)$ \\
\hline $\mathrm{C} 1-\mathrm{C} 2$ & $1.497(3)$ & $\mathrm{C} 7-\mathrm{H} 7$ & 0.9300 \\
\hline $\mathrm{C} 2-\mathrm{C} 3$ & $1.524(3)$ & $\mathrm{C} 8-\mathrm{C} 9$ & $1.377(3)$ \\
\hline $\mathrm{C} 2-\mathrm{H} 2 \mathrm{~A}$ & 0.9700 & $\mathrm{C} 8-\mathrm{H} 8$ & 0.9300 \\
\hline $\mathrm{C} 2-\mathrm{H} 2 \mathrm{~B}$ & 0.9700 & $\mathrm{C} 9-\mathrm{H} 9$ & 0.9300 \\
\hline $\mathrm{O} 5-\mathrm{Cl1}-\mathrm{O} 4$ & $110.73(15)$ & $\mathrm{N} 1-\mathrm{C} 3-\mathrm{C} 2$ & $109.89(18)$ \\
\hline $\mathrm{O} 5-\mathrm{C} 11-\mathrm{O} 6$ & $110.28(13)$ & $\mathrm{C} 4-\mathrm{C} 3-\mathrm{C} 2$ & $113.42(18)$ \\
\hline $\mathrm{O} 4-\mathrm{C} 11-\mathrm{O} 6$ & $109.36(13)$ & $\mathrm{N} 1-\mathrm{C} 3-\mathrm{H} 3$ & 107.5 \\
\hline $\mathrm{O} 5-\mathrm{Cl} 1-\mathrm{O} 3$ & $108.97(12)$ & $\mathrm{C} 4-\mathrm{C} 3-\mathrm{H} 3$ & 107.5 \\
\hline $\mathrm{O} 4-\mathrm{Cl} 1-\mathrm{O} 3$ & $108.21(12)$ & $\mathrm{C} 2-\mathrm{C} 3-\mathrm{H} 3$ & 107.5 \\
\hline $\mathrm{O} 6-\mathrm{C} 11-\mathrm{O} 3$ & $109.25(12)$ & $\mathrm{C} 5-\mathrm{C} 4-\mathrm{C} 9$ & $119.0(2)$ \\
\hline $\mathrm{C} 1-\mathrm{O} 1-\mathrm{H} 1$ & 109.5 & $\mathrm{C} 5-\mathrm{C} 4-\mathrm{C} 3$ & $122.6(2)$ \\
\hline $\mathrm{C} 3-\mathrm{N} 1-\mathrm{H} 1 \mathrm{~A}$ & 109.5 & $\mathrm{C} 9-\mathrm{C} 4-\mathrm{C} 3$ & $118.5(2)$ \\
\hline $\mathrm{C} 3-\mathrm{N} 1-\mathrm{H} 1 \mathrm{~B}$ & 109.5 & $\mathrm{C} 4-\mathrm{C} 5-\mathrm{C} 6$ & $120.1(3)$ \\
\hline $\mathrm{C} 3-\mathrm{N} 1-\mathrm{H} 1 \mathrm{C}$ & 109.5 & $\mathrm{C} 4-\mathrm{C} 5-\mathrm{H} 5$ & 120.0 \\
\hline $\mathrm{H} 1 \mathrm{~A}-\mathrm{N} 1-\mathrm{H} 1 \mathrm{~B}$ & 109.5 & $\mathrm{C} 6-\mathrm{C} 5-\mathrm{H} 5$ & 120.0 \\
\hline $\mathrm{H} 1 \mathrm{~A}-\mathrm{N} 1-\mathrm{H} 1 \mathrm{C}$ & 109.5 & $\mathrm{C} 7-\mathrm{C} 6-\mathrm{C} 5$ & $120.4(3)$ \\
\hline $\mathrm{H} 1 \mathrm{~B}-\mathrm{N} 1-\mathrm{H} 1 \mathrm{C}$ & 109.5 & $\mathrm{C} 7-\mathrm{C} 6-\mathrm{H} 6$ & 119.8 \\
\hline $\mathrm{O} 2-\mathrm{C} 1-\mathrm{O} 1$ & $123.8(2)$ & $\mathrm{C} 5-\mathrm{C} 6-\mathrm{H} 6$ & 119.8 \\
\hline $\mathrm{O} 2-\mathrm{C} 1-\mathrm{C} 2$ & $124.2(2)$ & $\mathrm{C} 8-\mathrm{C} 7-\mathrm{C} 6$ & $119.5(3)$ \\
\hline $\mathrm{O} 1-\mathrm{C} 1-\mathrm{C} 2$ & $111.91(19)$ & $\mathrm{C} 8-\mathrm{C} 7-\mathrm{H} 7$ & 120.2 \\
\hline $\mathrm{C} 1-\mathrm{C} 2-\mathrm{C} 3$ & $113.35(18)$ & $\mathrm{C} 6-\mathrm{C} 7-\mathrm{H} 7$ & 120.2 \\
\hline $\mathrm{C} 1-\mathrm{C} 2-\mathrm{H} 2 \mathrm{~A}$ & 108.9 & $\mathrm{C} 7-\mathrm{C} 8-\mathrm{C} 9$ & $120.5(3)$ \\
\hline $\mathrm{C} 3-\mathrm{C} 2-\mathrm{H} 2 \mathrm{~A}$ & 108.9 & $\mathrm{C} 7-\mathrm{C} 8-\mathrm{H} 8$ & 119.7 \\
\hline $\mathrm{C} 1-\mathrm{C} 2-\mathrm{H} 2 \mathrm{~B}$ & 108.9 & $\mathrm{C} 9-\mathrm{C} 8-\mathrm{H} 8$ & 119.7 \\
\hline $\mathrm{C} 3-\mathrm{C} 2-\mathrm{H} 2 \mathrm{~B}$ & 108.9 & $\mathrm{C} 4-\mathrm{C} 9-\mathrm{C} 8$ & $120.5(3)$ \\
\hline $\mathrm{H} 2 \mathrm{~A}-\mathrm{C} 2-\mathrm{H} 2 \mathrm{~B}$ & 107.7 & $\mathrm{C} 4-\mathrm{C} 9-\mathrm{H} 9$ & 119.7 \\
\hline $\mathrm{N} 1-\mathrm{C} 3-\mathrm{C} 4$ & 110.68 (19) & $\mathrm{C} 8-\mathrm{C} 9-\mathrm{H} 9$ & 119.7 \\
\hline
\end{tabular}

Hydrogen-bond geometry $\left(\AA,{ }^{\circ}\right)$

\begin{tabular}{lllll}
\hline$D-\mathrm{H} \cdots A$ & $D-\mathrm{H}$ & $\mathrm{H} \cdots A$ & $D \cdots A$ & $D-\mathrm{H} \cdots A$ \\
\hline $\mathrm{N} 1-\mathrm{H} 1 B \cdots \mathrm{O} 2$ & 0.89 & 2.13 & $2.773(3)$ & 128 \\
$\mathrm{C} 9-\mathrm{H} 9 \cdots \mathrm{O} 5^{\mathrm{i}}$ & 0.93 & 2.56 & $3.409(3)$ & 152 \\
$\mathrm{C} 3-\mathrm{H} 3 \cdots 5^{\mathrm{i}}$ & 0.98 & 2.57 & $3.370(3)$ & 139 \\
$\mathrm{C} 3-\mathrm{H} 3 \cdots 2^{\mathrm{ii}}$ & 0.98 & 2.58 & $3.286(3)$ & 129 \\
$\mathrm{~N} 1-\mathrm{H} 1 C \cdots \mathrm{O} 3^{\mathrm{iii}}$ & 0.89 & 2.05 & $2.892(3)$ & 158 \\
$\mathrm{~N} 1-\mathrm{H} 1 B \cdots 3^{\text {iv }}$ & 0.89 & 2.28 & $3.046(3)$ & 144 \\
$\mathrm{~N} 1-\mathrm{H} 1 A \cdots 6^{\mathrm{v}}$ & 0.89 & 2.13 & $2.979(3)$ & 159 \\
$\mathrm{O} 1-\mathrm{H} 1 \cdots \mathrm{O} 2^{\mathrm{vi}}$ & 0.82 & 2.41 & $3.046(2)$ & 135
\end{tabular}




\section{supporting information}

$\begin{array}{llllr}\mathrm{O} 1-\mathrm{H} 1 \cdots \mathrm{O} 4^{\text {vii }} & 0.82 & 2.35 & 3.048(3) & 143 \\ \mathrm{C} 8-\mathrm{H} 8 \cdots \mathrm{Cg} 1^{\text {viii }} & 0.93 & 2.79 & 3.688(3) & 162\end{array}$

Symmetry codes: (i) $-x+3 / 2, y-1 / 2, z$; (ii) $x-1 / 2, y,-z+1 / 2$; (iii) $x-1, y-1, z$; (iv) $x-1 / 2, y-1,-z+1 / 2$; (v) $x, y-1, z$; (vi) $x+1 / 2, y,-z+1 / 2$; (vii) $-x+2, y-1 / 2$, $-z+1 / 2$; (viii) $-x-1, y+1 / 2,-z+1 / 2$. 